\title{
Análisis del comportamiento del Modelo de Crecimiento de Gompertz en la predicción del crecimiento de la economía de Argentina, Bolivia, Chile y Perú
}

\author{
JUAN CARLOS PARRA MÁRQUEZ \\ Universidad del BIO-BIO (UBB), Facultad de Ciencias Empresariales, Avda.Collao 1202, 4081112 \\ Concepción, Chile.E-mail: jparra@ubiobio.cl
}

\section{RESUMEN}

El objetivo de este trabajo es predecir el crecimiento económico de Argentina, Bolivia, Chile y Perú a través del modelo de crecimiento biológico de Gompertz. Además, se propone un modelo que mejore la bondad de ajuste. Para para estimar los parámetros de ambos modelos y verificar su tasa de convergencia se utiliza los algoritmos de Gauss-Newton, Newton-Raphson y Levenberg-Marquardt. La elección del modelo de mejor aproximación se realiza según sus criterios de Akaike y Schwartz-Bayesian. Finalmente, los algoritmos se implementan en el software Matlab v. R2013b.

Palabras clave: Modelos de Crecimiento, AIC, Bootstrap, PIB.

\section{Study of Economic Growth of four Latin American Economies through the Gompertz's Growth Model}

\begin{abstract}
The aim of this paper is to predict the economic growth of Argentina, Bolivia, Chile, and Peru through the Gompertz's biological growth model. In addition, we propose a model that improves the goodness of fit. We use Gauss-Newton, Newton-Raphson, and Levenberg-Marquardt algorithms in order to estimate the parameters of both models and verify their rate of convergence. The best model specification is chosen according to its Akaike and Schwartz-Bayesian criteria. The algorithms are implemented in Matlab v. R2013b software.
\end{abstract}

Keywords: Growth Models, Akaike, Bootstrap, GDP.

Clasificación JEL: C01, C53, C87

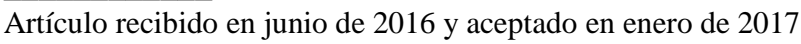

Artículo disponible en versión electrónica en la página www.revista-eea.net, ref. ə-35203 


\section{INTRODUCCIÓN}

El crecimiento económico es la sumatoria de muchas variables macroeconómicas que convergen para producir un efecto de estabilidad, bienestar y desarrollo para la sociedad que disfruta del mismo. Es así, que el crecimiento económico se evidencia cuando aumenta o disminuye el producto interno bruto (PIB) y, por tanto, si el PIB crece a un ritmo superior al del crecimiento de la población, se dice que el nivel de vida de la misma, aumenta. Si por el contrario, la tasa de crecimiento de la población es mayor que la tasa de crecimiento del PIB, se puede afirmar que el nivel de vida de la población está disminuyendo. De aquí, es que el crecimiento se puede medir en términos nominales o reales (es decir, descartando los efectos de la inflación). Si el PIB nominal aumentara a una tasa de crecimiento del $5 \%$ y la inflación fuese de una tasa del $4 \%$ en el mismo periodo, se puede indicar, en términos reales, que la tasa de crecimiento es del $1 \%$ que corresponderá al aumento real del PIB. Por ello, y por su importancia, es que la tasa de crecimiento económico se utiliza para realizar comparaciones entre distintas economías, o entre una economía y el grupo de países a la que pertenece.

Por otra parte, se tiene que en el tema de modelos de crecimiento existen diversos enfoques entre los cuales están los de índole biológica. En general, estos se analizan según ciertas propiedades de comportamiento del fenómeno en estudio siendo una de estas el clasificarlos tomando en cuenta el valor límite que pueda lograr la curva; por ello, se tiene curvas acotadas y no acotadas en su valor superior. Es así que Malthus (1803) propone un modelo interesante asociado a una curva exponencial e intenta describir el crecimiento de las poblaciones humanas con curvas no acotadas. Otro autor es Verhust (1845) que propone un modelo logístico que se caracteriza por una curva acotada y de forma sigmoidal; este modelo contempla que no sólo depende de su tamaño sino que además de la distancia a su cota superior. Otro ejemplo es el modelo de Gompertz (1825) que es muy similar al logístico pero que se diferencia en que el punto de inflexión se logra en la primera parte del ciclo de crecimiento y que, por ende, ha presentado un mejor ajuste de predicción en el análisis del crecimiento de ciertos seres vivos y, por lo tanto, se ha constituido en uno de los principales motivos por el cual se ha elegido como base inicial de este análisis. Otro autor, von Bertalanffy (1957) presenta un modelo para el análisis de poblaciones animales y en particular para el análisis de poblaciones de peces; como describe Carmen Sanchez (2013). Esta autora señala que dicha curva es una adaptación del modelo logístico y que se considera la existencia de una cota superior en el crecimiento que, en teoría, podría ser alcanzada.

En síntesis, el tema de estudio lo constituye el análisis del comportamiento de la curva estimada del PIB según sea su ajuste a la ecuación de crecimiento de Gompertz, en su versión de modelo de difusión, y que por medio del uso de algoritmos computacionales se pueda obtener la mejor estimación de los 
parámetros de dicha curva de predicción. Además, comparar estos resultados con los obtenidos con una propuesta propia de modelo de predicción.

Finalmente, otro aspecto a considerar es la transformación de modelos determinísticos de crecimiento en modelos heurísticos (denominados generalmente de difusión). Estos últimos corresponden a modelos a los cuales se les incorpora un término aleatorio (típicamente de distribución normal, con media 0 y varianza $\sigma^{2}$ ) que permite transformar el modelo determinístico en estocástico.

\section{ANTECEDENTES TEÓRICOS}

Las teorías del crecimiento económico estudian qué variables determinan el crecimiento de la economía al largo plazo y las políticas que deben impulsarse para estimular dicho crecimiento. Ahora bien, la historia del crecimiento económico es tan larga como la historia del pensamiento económico. Los primeros analistas económicos, como Adam Smith (1776), David Ricardo (1817) o Thomas Malthus (1803), estudiaron el tema del crecimiento e introdujeron conceptos fundamentales tales como el de rendimientos decrecientes y su relación con la acumulación de capital físico o humano, la relación entre el progreso tecnológico y la especialización del trabajo o el enfoque competitivo, como herramientas para el análisis de equilibrio dinámico.

Según diversos autores, como Corbo y Vergara (1992), señalan que pueden ser múltiples los factores que permiten explicar el crecimiento económico, siendo algunos de estos: trabajo, capital, capital humano, recursos naturales y tecnología, entre otros. Otros conceptos importantes para entender el crecimiento económico son: Las teorías del crecimiento económico, del ciclo económico y del desarrollo económico.

Las teorías del crecimiento económico se relacionan con el crecimiento de la producción de pleno empleo. Estas tienen sus primeros postulados a partir de Adam Smith, por el año 1776, hasta las más actuales y que explican su comportamiento a lo largo del tiempo. Por otra parte, el crecimiento económico es típicamente caracterizado por un comportamiento cíclico, que según señalan algunos analistas como Hawtrey y Tinbergen (1956), es un ciclo con una media aproximada de ocho años.

Adicionalmente, el tema de crecimiento económico posee una amplia literatura, tanto teórica como empírica. Los modelos teóricos, en general, tienen como objetivo el estudiar qué variables intervienen en el crecimiento económico. Por el contrario, los estudios empíricos enfrentan el problema de analizar aquellas variables de largo plazo que influyen en el corto plazo. El desarrollo teórico sobre los factores determinantes del crecimiento muestran su relevancia con los modelos neoclásicos desarrollados por Solow (1956); este trabajo se constituyó en el paradigma de la teoría e investigación empírica del crecimiento económico. Sin embargo, surge una creciente disconformidad en este campo porque algunas 
predicciones teóricas, como la hipótesis de convergencia, no se cumple claramente y es así que se dinamizan los planteamientos, del modelo anterior, a partir de los trabajos desarrollados por Cass (1965) y Koopmans (1965) que utilizan el método de optimización inter-temporal de Ramsey para exponer el comportamiento tanto del ahorro como del consumo. Los modelos neoclásicos indican que la existencia de rendimientos decrecientes para el capital y el trabajo implican que el crecimiento, a través de la acumulación de capital per cápita, tiende a un estado estacionario; por ello, los neoclásicos concluyen que el crecimiento económico y los aumentos de productividad, en el largo plazo, son objeto de los cambios tecnológicos y que estos son exógenos a sus modelos. Aquí es importante el concepto de innovación introducido por Schumpeter (1957) por su relevancia en el crecimiento. Según este autor, se tiene la existencia de dos situaciones de crecimiento, un estado de no crecimiento, o circuito económico, y un estado de crecimiento, la evolución. El paso del primer estado al segundo se presenta por medio de la innovación y esta última, según señala, corresponde al motor del crecimiento. En este contexto, se plantea el progreso tecnológico exógeno que corresponde a aquel progreso tecnológico que no explica las causas ni los orígenes del progreso tecnológico sino que simplemente asumen que este se da en forma exógena. No se debe dejar de mencionar que el modelo de Solow mantuvo su vigencia por aproximadamente tres décadas y que la base de su planteamiento fue que el crecimiento de largo plazo es exógeno lo que limitaba el rol de las políticas económicas para mejorarlo; es así que los modelos desarrollados por Romer (1986) y Lucas (1988) potencian la teoría del crecimiento con sus propios planteamientos; estos modelos son llamados de crecimiento endógeno, puesto que buscan explicar que el crecimiento, en el largo plazo, no es debido a factores exógenos; es decir, es endógeno. Con ello, el modelo de Romer, explica el crecimiento económico descartando los rendimientos decrecientes del capital a través de introducir externalidades positivas en la acumulación de capital a nivel agregado. Este modelo supone que el conocimiento y la tecnología deben ser considerados un bien público y, por lo tanto, que al darse un nuevo conocimiento tecnológico, en una empresa, éste se difunde a toda la economía. Estas externalidades permiten que el modelo neoclásico se convierta en un modelo lineal semejante al desarrollado por Rebelo (1991). Este tipo de modelo permite, en el largo plazo, que no existan rendimientos decrecientes del capital que deriva en una tasa de crecimiento positivo y constante. Entonces, como producto de estos enfoques (endógeno y exógeno), los países podrían presentar tasas de crecimiento sostenidas en el tiempo, basadas en su capacidad de innovar, y sin que se dé la anunciada convergencia de la teoría neoclásica. Una consecuencia de lo anterior era que ahora la teoría ofrecía dos visiones sobre el crecimiento que debían ser contrastadas con la evidencia internacional. De manera paralela, con el resurgimiento de la teoría del crecimiento, se publicaba una base de datos sobre 
cuentas nacionales para más de 130 países con información desde 1960 a 1985. Dicha base de datos tenía la ventaja de ser comparable internacionalmente, dado que los agregados macroeconómicos ahí publicados estaban expresados en la misma unidad (el dólar). El primer estudio conocido en utilizar dichos datos, para un análisis sobre los determinantes del crecimiento, fue el de Robert Barro (1991), el cual dio origen a lo que hoy se conoce como literatura empírica del crecimiento.

Más recientemente, los modelos de crecimiento endógeno plantearon la posibilidad de que el comercio internacional permite a una economía crecer continuamente puesto que también existe la posibilidad de generar ganancias dinámicas, Lee (1995), Coe y Helpman (1993); es decir, plantean desplazamientos en las posibilidades de producción. La idea es que las tasas de crecimiento de los países pueden estar relacionadas a través del comercio internacional y de los conocimientos incorporados en los bienes involucrados en el proceso cuando ocurre un desborde de conocimiento al comerciar con países más avanzados tecnológicamente. Esto sucedería esencialmente porque el conocimiento es un bien no rival cuya utilización y difusión puede ocasionar efectos secundarios y aumentos de productividad. En este contexto, Edwards (1997) utiliza distintos indicadores de apertura comercial y encuentra de forma clara un efecto positivo en el crecimiento en una muestra de 93 países. Por otra parte, en lo que respecta al capital humano, Barro y Lee (2010) construyen indicadores que miden los años de educación de la población mayor de 15 años para los años 1950-2010. Ellos encuentran, en una muestra de 146 países, que la acumulación de años de educación tiene un efecto positivo en el crecimiento económico.

Por ejemplo, en Chile Coeymans (1999) realiza un estudio sobre los determinantes de la productividad utilizando dos modelos de productividad agregada endógena. Aquí encuentra que los shocks externos tienen efectos en el corto plazo y generan ciclos económicos; esto debido a que los shocks hacen que los agentes económicos reduzcan la demanda agregada y se genere un aumento a las tasas de interés; lo que origina caídas en la productividad como consecuencia de un menor acceso a capital de trabajo y una menor utilización de los factores productivos. Como shocks externos se identifican los cambios a los servicios financieros de la deuda, los términos de intercambio y las caídas en las reservas internacionales. El estudio encuentra que la apertura de la economía chilena tuvo un efecto positivo en el crecimiento en el largo plazo.

Finalmente, Corbo y Vergara (1992), señalan que debido a la relevancia del tema el Instituto de Economía de la Universidad Católica, con el auspicio del Banco Central de Chile, decide organizar una conferencia sobre los principales determinantes del crecimiento económico entre los días 8 y 9 de junio de 1992. En dicho artículo, se comenta algunos de los resultados de dicho evento, entre 
los cuales se pueden destacar el comentario sobre el artículo de Stanley Fisher que señala que dicho autor encuentra que, después de controlar por la acumulación de factores, queda una parte importante del crecimiento del producto que es explicada por la estabilidad de las políticas macroeconómicas, dicha estabilidad afecta directamente el crecimiento vía la eficiencia en el uso de los factores e, indirectamente, a través de la tasa de inversión. También se señala, Felipe Larraín y Rodrigo Vergara, en su hipótesis relacionada con el crecimiento económico, este se ve afectado por un factor estructural del país: la distribución del ingreso; en palabras simples, que una mayor desigualdad en la distribución del ingreso retarda el proceso del crecimiento de los países. Ahora bien, es interesante que la desigualdad tiene un efecto ambiguo sobre el crecimiento, Aghion et al. (1999) señalan que ésta puede ser beneficiosa si las diferencias de ingreso representan incentivos para los que tienen menos; además, también podría fomentar el ahorro y posibilitar la inversión. Por otro lado, puede ser perjudicial si genera una mayor volatilidad macroeconómica. Otro fundamento, por el cual la desigualdad afecta negativamente al crecimiento, es aquel en donde las sociedades menos tolerantes a la desigualdad pueden terminar obstaculizando procesos de desarrollo en los que inicialmente se genera una inequitativa distribución del ingreso. Alesina y Rodrik (1994) presentan un modelo donde la desigualdad en la distribución del ingreso tiende a fomentar políticas que retardan el crecimiento bajo la forma de mayores impuestos al capital y muestran evidencia empírica donde la desigualdad medida a través del coeficiente de Gini, aplicado a la distribución de la tierra, impacta negativamente sobre la tasa de crecimiento de largo plazo.

\section{METODOLOGÍA}

Para realizar este estudio, primeramente se realiza un análisis del modelo de crecimiento de Gompertz; ello de tal manera de visualizar como se ajusta al crecimiento del PIB y luego se aplica la nueva propuesta de modelo para luego confrontarlos bajo algún criterio estadístico de comparación (por ejemplo, índice de Akaike). Para estimar los parámetros se aplica máxima verosimilitud al modelo de tal forma de que estos sean los que minimicen los valores del PIB estimado con los valores reales.

Ahora bien, el PIB es la variable de la economía en estudio puesto que esta es la de mayor uso en el análisis y comparación de las economías de las naciones y, además, permite tomar decisiones económicas estratégicas permitiendo, a partir de esta, ajustar otras variables económicas.

Posteriormente, se busca información de algoritmos que reflejen la orientación o estrategias de estos para la determinación de los valores de los parámetros de ajuste de un modelo a un determinado conjunto de datos obtenidos de alguna muestra determinada. 
En lo referente a los datos, los países de análisis han elegidos bajo el criterio de vecindad de tal manera que se pueda tener un cierto comportamiento de la región local y por el intercambio comercial existente entre ellos. Dichos datos fueron obtenidos a partir de la publicación en la página Web del Banco Mundial para así contar con datos estandarizados entre los países y que estos puedan ser consistentemente comparables; se debe señalar que estos han sido contrastados con los publicados por la CEPAL (CEPALSTAT, 2016) siendo similares salvo algunos casos en que la diferencia es sólo de algunos cientos de dólares. Por otra parte, dicha fuente tiene validación internacional y se basa en información fidedigna entregada por las naciones y que permite comparar las naciones en sus niveles de crecimiento.

Finalmente, la implementación se realiza en Matlab v. R2013b siendo éste un software de uso masivo y de fácil presentación algorítmica. La determinación de los valores iniciales de los procesos algorítmicos se realiza mediante la creación de aplicaciones iterativas que realizan una búsqueda aproximada de valores adecuados para tal efecto. Este proceso se vio favorecido por la capacidad de Levenberg-Marquardt que fue el único de los algoritmos implementados que, con una aproximación simple a dicha solución, convergió a la solución más rápido que los otros. Adicionalmente, se implementan otros algoritmos que permitieran, por ejemplo, verificar si el comportamiento de los residuos es del tipo normal.

\section{MODELOS DE CRECIMIENTO DE ESTUDIO}

\subsection{Modelo de Crecimiento de Gompertz}

La ecuación diferencial que da origen al modelo de Gompertz es:

$$
\frac{d y(t)}{d t}=K y(t) \ln \left(\frac{A}{y(t)}\right)
$$

donde

$y(t)$ corresponde al tamaño de la población en el tiempo t.

A valor máximo de crecimiento.

$K$ tasa de crecimiento de la curva.

Se supone que $K>0$. Ahora bien, luego de un proceso matemático de la expresión anterior, se llega finalmente a la expresión del modelo de crecimiento de Gompertz cuya expresión final es:

$$
y(t)=A e^{-B e^{-K t}}
$$

donde

$y(t)$ corresponde al tamaño de la población en el tiempo $t$. 
A valor máximo de crecimiento.

$B$ valor que permite que la curva se desplace a la izquierda o derecha según sean los datos.

$K$ tasa de crecimiento de la curva.

Este modelo es muy utilizado para el estudio de la ley de mortalidad humana que fue propuesto originalmente por Benjamín Gompertz en 1925 y que lo presentó con el objetivo de predecir el comportamiento de dicha ley.

A partir de lo anterior, el Modelo Gompertz determinístico (o de difusión), tiene la expresión:

$$
\hat{y}(t)=A e^{B e^{-K t}}+\varepsilon
$$

donde $\varepsilon \sim N\left(0, \sigma^{2}\right)$ corresponde a los residuos de la estimación; es decir $\varepsilon=y$ $\hat{y}$, siendo ŷ el valor estimado del PIB; $A, B, K$ corresponden a los parámetros estimados de ajuste, $t$ es la variable de tiempo (periodo de datos observados del PIB de análisis: 1962-2014).

\subsection{Modelo Propuesto}

Dado todo el proceso de análisis, tanto el estructural y de comportamiento de varios modelos analizados en este estudio y otros (Modelos analizados Bertalanffy, Logístico y Brody) como el de las curvas de comportamiento del PIB de los países analizados, se ha logrado cierta experiencia en los aspectos generales de dichas conductas por lo que se planteó un modelo sobre la base de lograr una mejor aproximación del proceso cíclico que presentan los datos muestrales incorporando la expresión del movimiento armónico simple con amplitud variable en t en la función exponencial y el valor máximo crecimiento variable también por t; es así que, luego de varios intentos empíricos, la propuesta de modelo es la siguiente:

$$
y(t)=\left(A t^{m}\right) e^{-B t \cos (w t+\theta)}+\varepsilon
$$

donde las variables corresponden a:

$y(t)$ tamaño de la población en el tiempo $t$.

$t$ variable tiempo.

$A t^{m}$ valor máximo de crecimiento (variable al depender de $t$ ).

$B t$ amplitud (de valor variable por estar afecta a $t$ ) dentro de un movimiento armónico.

$w$ frecuencia de la curva para un movimiento armónico.

$\theta$ desfase del movimiento armónico.

Por tanto, esta propuesta no se basa en ningún proceso analítico en particular sino sólo es producto del análisis del comportamiento señalado anteriormente y 
de ajustar los modelos de crecimiento biológico con un movimiento armónico y con el concepto de variabilidad de los coeficientes que los otros modelos consideran constantes.

\section{PIB DE PAÍSES EN ANÁLISIS}

En lo referido a los datos observados, los países elegidos, como se ha mencionado en párrafos anteriores, corresponden a Argentina, Bolivia, Chile y Perú. Estos presentan un comportamiento de crecimiento (a partir del PIB) entre los años 1962 y 2014 como se muestra en Figura 1.

Figura 1

Gráfica resumen de los PIB de los países a modelar (Argentina, Bolivia, Chile y Perú)

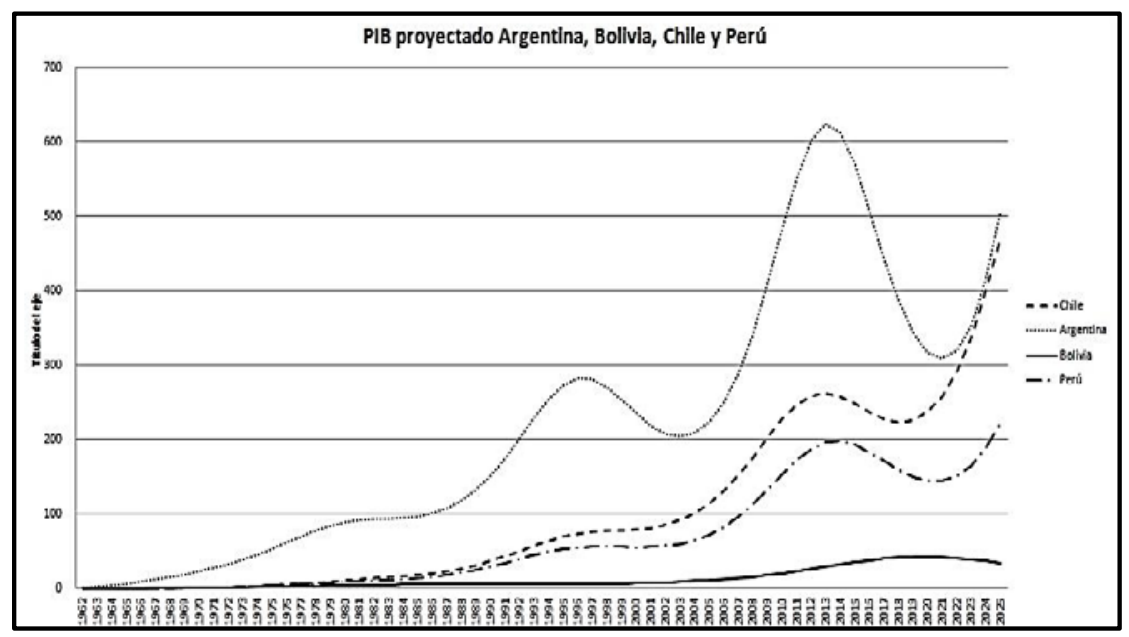

Fuente: Elaboración propia.

Se deber reiterar que los países han sido elegidos para el estudio bajo el criterio de vecindad así como por la incidencia directa que tienen con Chile en el tema de intercambio comercial; en particular, por el gran impacto, en la última década para Chile, en el tema del incremento en el intercambio turístico (objetivo indirecto de este estudio) y que han constituido un aporte no menor al PIB chileno. Además, se debe señalar que el estudio del comportamiento de los demás países de la región, tanto los andinos como los de la costa atlántica, está en curso y sobre la base de los resultados modelados en este estudio.

\section{MAXIMA VEROSIMILITUD}

El método de máxima verosimilitud se puede considerar como el de mayor utilización y, por ende, el clásico de los clásicos. Es así como la mayor parte de los estadísticos lo recomiendan por obtener resultados suficientes y consistentes. 
Ahora bien, si se tiene una variable aleatoria Y con función de distribución $\mathrm{N}\left(\mathrm{h}(\mathrm{t} ; \theta), \sigma^{2}\right)$ y sean $\mathrm{y} 1, \mathrm{y} 2, \ldots$, yn los datos obtenidos de una muestra recogidos mediante algún experimento del fenómeno a estudiar, se puede presentar el modelo estocástico:

$$
y_{i}=h\left(t_{i} ; \theta\right)+\varepsilon
$$

donde

$y_{i} \quad$ corresponde a las observaciones en el instante $t_{i}$.

$h$ es algún modelo matemático como el de Bertalanffy, el Gompertz o cualquier otro.

$\theta \quad$ corresponde al vector de parámetros del modelo.

$t_{i} \quad$ la variable tiempo.

$\varepsilon \quad$ corresponde al error aleatorio con distribución $N\left(0, \sigma^{2}\right)$.

El objetivo es el determinar los parámetros, $\theta$, para lo cual se hace uso del método de máxima verosimilitud. Para ello, se define la función de verosimilitud siguiente:

$$
L(\theta)=\prod_{i=1}^{n} \frac{1}{\sqrt{2 \pi \sigma^{2}}} e^{-\frac{\left(y_{i}-h\left(t_{i} ; \theta\right)\right)^{2}}{2 \sigma^{2}}}
$$

$\mathrm{Al}$ aplicar logaritmo neperiano se tiene:

$$
\ln \left(L\left(\theta, \sigma^{2}\right)\right)=-n \ln \left(\sqrt{2 \pi \sigma^{2}}\right)-\frac{1}{2 \sigma^{2}} \sum_{i=1}^{n}\left(y_{i}-h\left(t_{i} ; \theta\right)\right)^{2}
$$

que corresponde a la expresión a maximizar mediante este método.

Para maximizar esta última expresión, se debe minimizar el único término variable de la misma; es decir:

$$
\min _{\theta} \sum_{i=1}^{n}\left(y_{i}-h\left(t_{i} ; \theta\right)\right)^{2}
$$

Para resolver este problema se hace uso de algoritmos iterativos de minimización que hacen uso de, por ejemplo, el método de mínimos cuadrados. Este último, para problemas no lineales, surge de modelos matemáticos que permiten el estudio del comportamiento de sistemas económicos, sociales y físicos donde se requiere obtener aproximaciones a formulaciones más estándares o de fácil manipulación para realizar predicciones del valor futuro de las variables involucradas y cuyo comportamiento es no lineal.

El objetivo es encontrar el mínimo de la suma de los cuadrados de $n$ funciones no lineales; ello es equivalente a: 


$$
\min f(x)=\frac{1}{2} \sum_{i=1}^{n} r_{i}(x)^{2}=\frac{1}{2}\|r(x)\|^{2}
$$

donde $r(x)$ es el vector de residuos; en este caso:

$$
r(\theta)=\left(y_{i}-h\left(t_{i} ; \theta\right)\right)
$$

En general los problemas a resolver pertenecen al mundo real en los cuales no es factible encontrar una solución exacta por lo que se requiere una aproximación aceptable de la misma.

\section{CRITERIO DE INFORMACIÓN DE AKAIKE}

Existe una gran variedad de criterios de información $(C I)$, correspondientes a los criterios AIC y SBC, que se pueden representar mediante la siguiente expresión (Sclove, 1987):

$$
C I_{j}=-2 \ln \left(L\left(\hat{\theta}_{j}\right)\right)+k_{j} A(n)+B(j, n)
$$

donde

$C I_{j} \quad$ criterio de información del modelo $j$.

$n \quad$ tamaño de la muestra.

$\ln \left(L\left(\theta_{j}\right)\right)$ logaritmo neperiano de la verosimilitud maximizado para el modelo $j$ y de parámetros $\theta_{j}$.

$k_{j} \quad$ número de parámetros del modelo $j$.

$A(n) \quad$ costo de ajustar un parámetro adicional.

$B(j, n) \quad$ término adicional que depende del criterio y del modelo $j$.

Esta ecuación fue modificada (Yang y Yang, 2007) a la forma:

$$
C I_{j}=-2 \ln \left(L\left(\hat{\theta}_{j}\right)\right)+k_{j}(A(n)+C)+B(j, n)
$$

donde $C$ es una constante y que representa algunos de los principales criterios encontrados en la literatura científica y que permite clasificar a $C I_{j}$ en función de su pertenencia a la familia AIC (Akaike, 1974) o a la familia SBC o BIC (Schwartz, 1978).

Caballero (2011) señala que para series temporales y modelos de regresión se utiliza una corrección al AIC denominado AIC corregido, AICC, y es propuesto con el objetivo de reducir el sesgo del AIC (Sugiura, 1978). Además, Hurvich y Tsai $(1989,1991)$ verificaron que el AICC no sólo generaba una reducción considerable del sesgo sino que además mejora la selección del modelo más adecuado en tamaños muestrales pequeños (como es el caso de los PIB de este trabajo). Por lo tanto, el AICC es de uso cuando las muestras son pequeñas o 
cuando el número de parámetros es moderado o grande con respecto al tamaño de la muestra.

Por otra parte, Burnham y Anderson (2002), entre otros, recomiendan la utilización de esta corrección cuando además se cumpla la condición $n / \mathrm{k}_{\mathrm{j}}<40$; como es el caso de este trabajo. Para el caso de tamaños muestrales de tamaño grande, el valor de AICC es muy similar a AIC.

Para el criterio SBC Draper (1995) sugiere la modificación de la expresión a la forma, para modelos de tamaño muestral pequeño:

$$
D B I C=-2 \ln \left(L\left(\hat{\theta}_{j}\right)\right)+k_{j}(\ln (n)-\ln (2 \pi))
$$

Los coeficientes a utilizar en este trabajo se presentan en Tabla 1 (Caballero, 2011).

Tabla 1

Valores coeficientes de Criterios de Información

\begin{tabular}{|c|c|c|c|c|}
\hline & & $\boldsymbol{A}(\boldsymbol{n})$ & $\boldsymbol{C}$ & $\boldsymbol{B}(\mathbf{j}, \boldsymbol{n})$ \\
\hline Familia AIC & AICC (Sugiura, 1978) & 0 & 2 & $\frac{2\left(k_{j}+1\right)\left(k_{j}+2\right)}{n-k_{j}-2}$ \\
\hline Familia BIC & DBIC (Draper, 1995) & $\ln n$ & $-\ln (2 \pi)$ & 0 \\
\hline
\end{tabular}

Fuente: Elaboración propia.

\section{INTERVALO DE CONFIANZA PERCENTIL BOOTSTRAP}

El intervalo de confianza Bootstrap percentil, con un (1- $\alpha$ ) por ciento de confianza, para $\theta$ es:

$$
\theta:\left[\hat{\theta}_{\left(\frac{\alpha}{2}\right) B}^{*} ; \hat{\theta}_{\left(1-\frac{\alpha}{2}\right) B}^{*}\right]
$$

Para estos extremos se utilizan los cuantiles $(\alpha / 2)$ y $(1-\alpha / 2)$ de la muestra Bootstrap. El proceso se inicia generando $B$ conjuntos independientes de muestras Bootstrap.

\section{ESTIMACIÓN DE PARÁMETROS DEL PIB DE ARGENTINA, BOLIVIA, CHILE Y PERÚ}

Se aplica ambos modelos a cada serie de datos mediante los algoritmos iterativos (Levenberg-Marquardt, Gauss-Newton y Newton-Raphson) de tal forma de, adicionalmente, verificar el comportamiento de estos últimos. Con ello, se obtienen los parámetros de ajuste de la expresión matemática del modelo y con estos se procede a estimar el comportamiento del PIB de los años 2011, 
2012, 2013 y 2014 para cada país según el modelo elegido por su mejor comportamiento bajo el criterio AIC y BIC.

En Tabla 2 se presentan los coeficientes obtenidos para el modelo Gompertz, aplicado a los cuatro países considerados, y sus respectivos intervalos de confianza Bootstrap Percentil al 90\% de confianza.

Tabla 2

Valores de parámetros estimados modelo Gompertz e Intervalos de confianza para estimar PIB de Argentina, Bolivia, Chile y Perú

\begin{tabular}{|l|c|c|c|}
\hline & A & B & K \\
\hline Chile & 0.8150 & 2.1503 & -0.0194 \\
\hline Intervalo de confianza & {$[0.0066,3.4625]$} & {$[0.9386,6.6134]$} & {$[-0.0305,-0.0090]$} \\
\hline Argentina & 1048 & -4.4054 & 0.0288 \\
\hline Intervalo de confianza & {$[375.9036$,} & {$[-12.4236,-4.1724]$} & {$[0.0052,0.0611]$} \\
\hline Bolivia & $6223815.1139]$ & 0.9025 & -0.0260 \\
\hline Intervalo de confianza & {$[0.0005,1.7644]$} & {$[0.2654,7.7340]$} & {$[-0.0449,-0.0056]$} \\
\hline Perú & 2.5204 & 1.0777 & -0.0268 \\
\hline Intervalo de confianza & {$[0.0303,4.9615]$} & {$[0.5529,5.1162]$} & {$[-0.0370,-0.0098]$} \\
\hline
\end{tabular}

Fuente: Elaboración propia.

En Tabla 3 se presenta los resultados de aplicar los algoritmos de ajuste para los parámetros del nuevo modelo propuesto y sus respectivos intervalos de confianza Bootstrap Percentil al 90\% de confianza.

Tabla 3

Valores de parámetros estimados del nuevo modelo propuesto y sus respectivos intervalos de confianza para estimar PIB de Argentina, Bolivia, Chile y Perú

\begin{tabular}{|c|c|c|c|c|c|}
\hline & $A$ & $B$ & $w$ & $\theta$ & $m$ \\
\hline Chile & 0.7428 & -0.0089 & -0.3653 & -0.1306 & 1.5928 \\
\hline Intervalo de confianza & $\begin{array}{c}{\left[\begin{array}{c}0.4062 \\
18.0853]\end{array}\right.}\end{array}$ & $\begin{array}{c}{[-3.3238} \\
-0.0076]\end{array}$ & $\begin{array}{l}{[-0.4134,} \\
-0.0002]\end{array}$ & $\begin{array}{c}{[-14.1277,} \\
-1.6696]\end{array}$ & $\begin{array}{c}{\left[\begin{array}{c}0.0340 \\
1.7699\end{array}\right]}\end{array}$ \\
\hline Argentina & 0.0044 & -0.0057 & 0.4648 & 3.0830 & 2.6972 \\
\hline Intervalo de confianza & $\begin{array}{c}0.0033, \\
1.2218]\end{array}$ & $\begin{array}{c}-3.2657 \\
-0.0054]\end{array}$ & $\begin{array}{c}0.0004 \\
0.5195]\end{array}$ & $\begin{array}{c}{\left[\begin{array}{c}0.9252 \\
17.2651]\end{array}\right.}\end{array}$ & $\begin{array}{r}{\left[\begin{array}{c}1.1109 \\
2.7704\end{array}\right]}\end{array}$ \\
\hline Bolivia & 0.0004 & -0.1188 & 0.0384 & 2.0209 & 3.8278 \\
\hline Intervalo de confianza & $\begin{array}{c}0.0000 \\
0.0065]\end{array}$ & $\begin{array}{c}{[-1.9349} \\
-0.0390]\end{array}$ & $\begin{array}{c}0.0032, \\
0.0721]\end{array}$ & $\begin{array}{c}0.6229 \\
4.3691]\end{array}$ & $\begin{array}{c}\text { [ } 2.3426 \\
9.5857 \text { ] }\end{array}$ \\
\hline Perú & 0.0117 & -0.0061 & 0.4233 & 4.5992 & 2.3339 \\
\hline Intervalo de confianza & $\begin{array}{c}0.0060 \\
0.0472]\end{array}$ & $\begin{array}{c}-0.0067, \\
0.0071]\end{array}$ & $\begin{array}{c}{[0.3962,} \\
0.4876 \text { ] }\end{array}$ & $\begin{array}{c}3.0177, \\
8.7214 \text { ] }\end{array}$ & $\begin{array}{r}\text { [ } 1.9471 \\
2.5137 \text { ] }\end{array}$ \\
\hline
\end{tabular}

Fuente: Elaboración propia. 


\subsection{Análisis de Chile}

En Tabla 2 se puede visualizar los coeficientes del modelo Gompertz aplicados a los cuatro países en estudio y sus intervalos de confianza. En particular, desde el punto de vista estadístico, los coeficientes para el PIB de Chile presentan valores cuyos intervalos de confianza no incluyen el valor cero; es decir, ninguno de los coeficientes se anularía; este comentario también es válido para los demás países y para ambos modelos.

Para Chile, utilizando los coeficientes obtenidos y presentados en Tabla 2 y Tabla 3 se obtienen las curvas de ajuste (Figura 2) para ambos modelos. La curva del PIB original ha sido montada sobre las curvas obtenidas de los modelos.

Figura 2

Curva del PIB chileno obtenida con el modelo Gompertz (a la izquierda) y modelo propuesto (a la derecha)
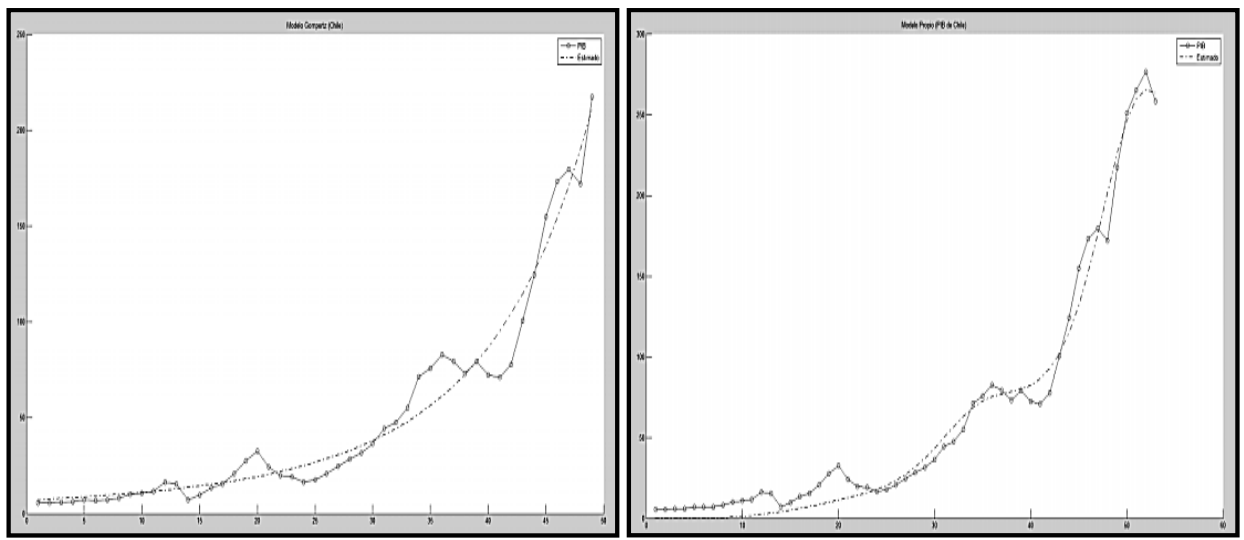

Fuente: Elaboración propia.

A simple vista, y gráficamente, se puede visualizar que el modelo propuesto (gráfico del lado derecho) se ajusta de mejor forma a la curva del PIB chileno evidenciándose mediante una curvatura de mejor aproximación a la original.

Ahora bien, es evidente el crecimiento del PIB chileno pero este no está exento de fluctuaciones que deben ser consideradas al momento de la toma de decisiones y que es de importante necesidad de pronosticar para los años próximos; por ende, se requiere una aproximación adecuada de la misma que permita apoyar las decisiones en los sectores productivos chilenos; en particular, en las inversiones relacionadas con el turismo.

Los análisis siguientes, para todos los casos, sólo se realizarán sobre la base de los resultados ajuste y aproximación de ambos modelos a los datos observados para, posteriormente, realizar una pequeña discusión de las tendencias aproximadas del PIB de los países involucrados. 


\subsection{Análisis de Argentina}

Para Argentina, se presenta en Tabla 2 los valores de los parámetros de ajuste del modelo Gompertz y en Tabla 3 los del nuevo modelo propuesto. Además, también se presentan los respectivos intervalos de confianza según Bootstrap.

Primeramente, los intervalos de confianza indican que ninguno de los parámetros se anula, tanto para el modelo propuesto como para el modelo Gompertz. Por otra parte, gráficamente las curvas de ajuste de ambos modelos se presentan en Figura 3 e indican que el modelo propuesto es se ajusta de mejor forma a la curva original.

\section{Figura 3}

Curva del PIB argentino y curva de tendencia obtenida con modelo Gompertz y modelo propuesto

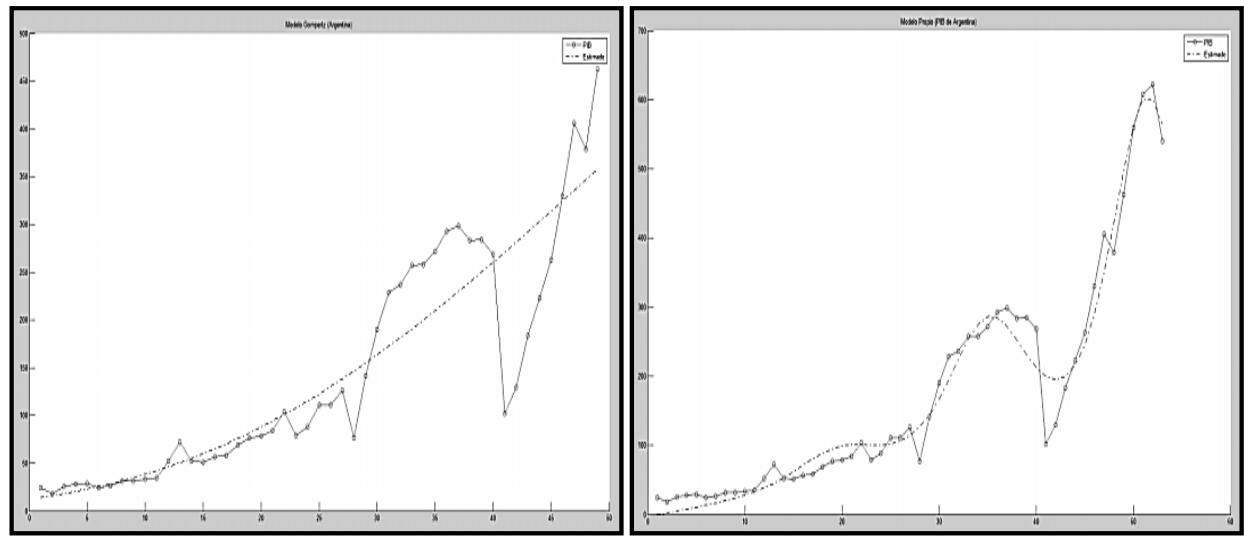

Fuente: Elaboración propia.

Entonces, se puede observar que el modelo propuesto se ajusta de mejor forma las oscilaciones que presenta la curva del PIB argentino; lo que no ha sido posible realizar mediante la curva obtenida a través del modelo Gompertz.

\subsection{Análisis de Bolivia}

Aplicando los dos modelos al PIB de Bolivia, se obtienen los resultados presentados en las Tabla 2 y 3 respectivamente. Para los intervalos de confianza de ambos modelos aplicados al PIB boliviano se tiene que los parámetros no incluyen el valor cero; por lo que ninguno se anularía. En Figura 4 se puede visualizar a simple vista que gráficamente nuevamente el modelo propuesto se ajusta de mejor forma. 
Figura 4

Curva de PIB de Bolivia y la curva de tendencia obtenida con el modelo Gompertz y modelo propuesto
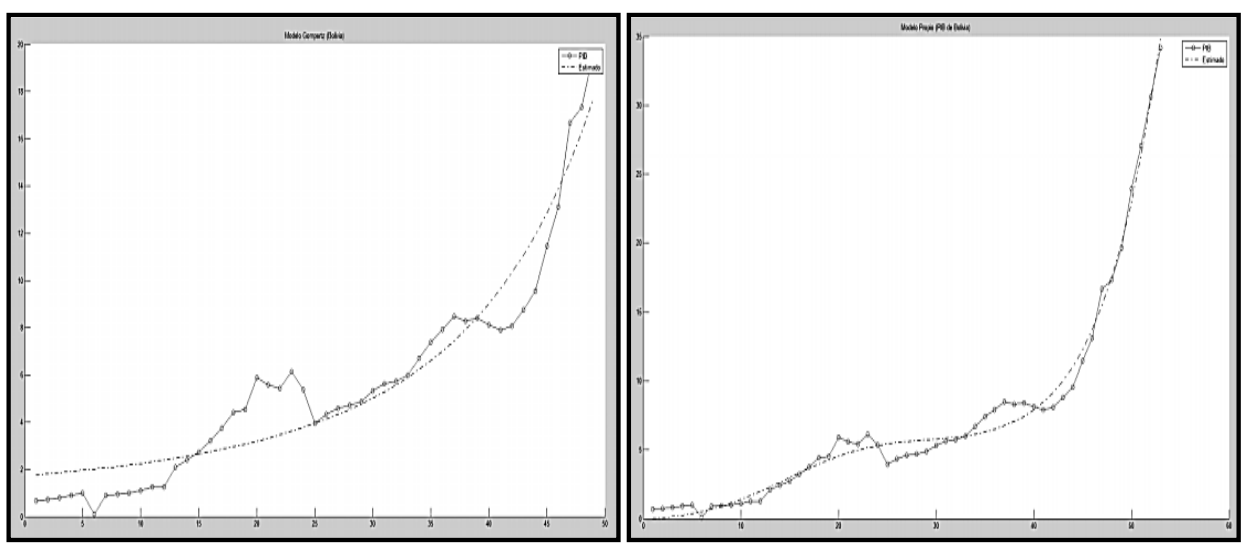

Fuente: Elaboración propia.

\subsection{Análisis de Perú}

Finalmente, para el PIB de Perú se obtienen los ajustes a los modelos también tabulados en Tabla 2 y 3 teniendo las mismas apreciaciones estadísticas que en los casos anteriores. En Figura 5 se puede también ver gráficamente que el modelo propuesto se ajusta de mejor forma a la curvatura de los datos originales.

Figura 5

Curva de PIB de Perú y la curva de tendencia obtenida con modelo Gompertz
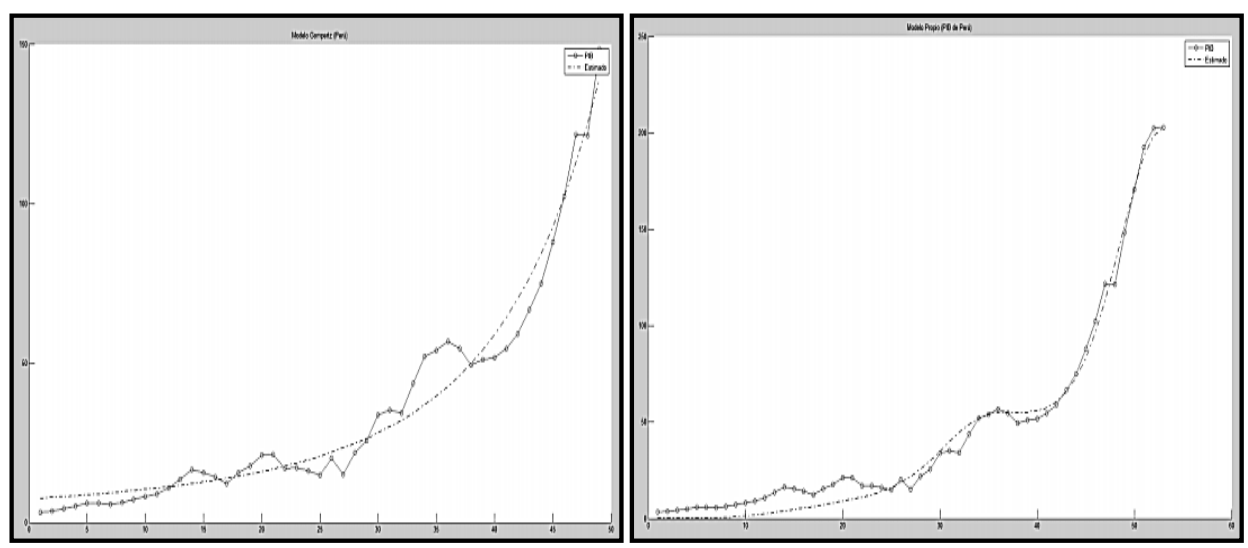

Fuente: Elaboración propia.

\subsection{Síntesis de resultados}

Primeramente, se puede señalar que observando la Tabla 4 que resume el 
indicador AIC y BIC para todos los casos se puede concluir que para todos los países considerados el modelo que mejor se ajusta a los datos observados son el nuevo modelo propuesto; ello, aunque su obtención demanda más tiempo de procesamiento computacional que el de Gompertz por poseer 5 parámetros a diferencia de los 3 de este último.

Tabla 4

AIC y SBC (BIC) del nuevo modelo propuesto

\begin{tabular}{|c|c|c|c|c|}
\hline \multirow{2}{*}{ País } & \multicolumn{2}{|c|}{ Modelo Propuesto } & \multicolumn{2}{c|}{ Gompertz } \\
\cline { 2 - 5 } & AIC & BIC & AIC & BIC \\
\hline Chile & 358.8893 & 357.1590 & 371.5047 & 370.7575 \\
\hline Argentina & 480.6805 & 478.9502 & 532.2651 & 531.5178 \\
\hline Bolivia & 125.5496 & 123.8193 & 170.6482 & 169.9009 \\
\hline Perú & 310.3558 & 308.6255 & 324.0907 & 323.3434 \\
\hline
\end{tabular}

Fuente: Elaboración propia.

Tanto el indicador AIC como el BIC corroboran lo anterior al poseer valores menores para el nuevo modelo.

El trabajo futuro a seguir es el comprobar si este último modelo se ajusta razonablemente a otras naciones para predecir su comportamiento.

Adicionalmente, se ha calculado el coeficiente de determinación $\mathrm{R}^{2}$ de cada modelo y para cada serie, según país. Los resultados se presentan en Tabla 5.

Tabla 5

Cálculo de $\mathrm{R}^{2}$ para todos los modelos aplicados a los cuatro países en estudio

\begin{tabular}{|c|c|c|c|c|}
\hline & Chile & Argentina & Bolivia & Perú \\
\hline Gompertz & 0.9643 & 0.8066 & 0.9139 & 0.967 \\
\hline Modelo Propuesto & 0,9751 & 0,9392 & 0,9691 & 0,9776 \\
\hline
\end{tabular}

Fuente: Elaboración propia.

Para ambas tablas se confirma, en todos los casos, las recomendaciones mediante de AIC y BIC indicando que el modelo propuesta presenta una aproximación mejorada con respecto a Gompertz.

En general, los modelos aportan una aproximación aceptable que permite visualizar la tendencia de la misma aunque se debe recordar que en la economía existen diversas variables que inciden en su comportamiento. Lo recomendable sería proyectar cada una de esas variables para obtener un análisis más exhaustivo.

Para el modelo elegido, en Figura 6, se puede visualizar gráficamente los resultados de este nuevo modelo ajustado a los PIB de los países en estudio, 
añadiendo una proyección de cada nación a 10 años; como un ejercicio de aproximación.

Figura 6

Curva aproximada de la tendencia futura del PIB de Argentina, Bolivia, Chile y Perú obtenida a través del nuevo modelo

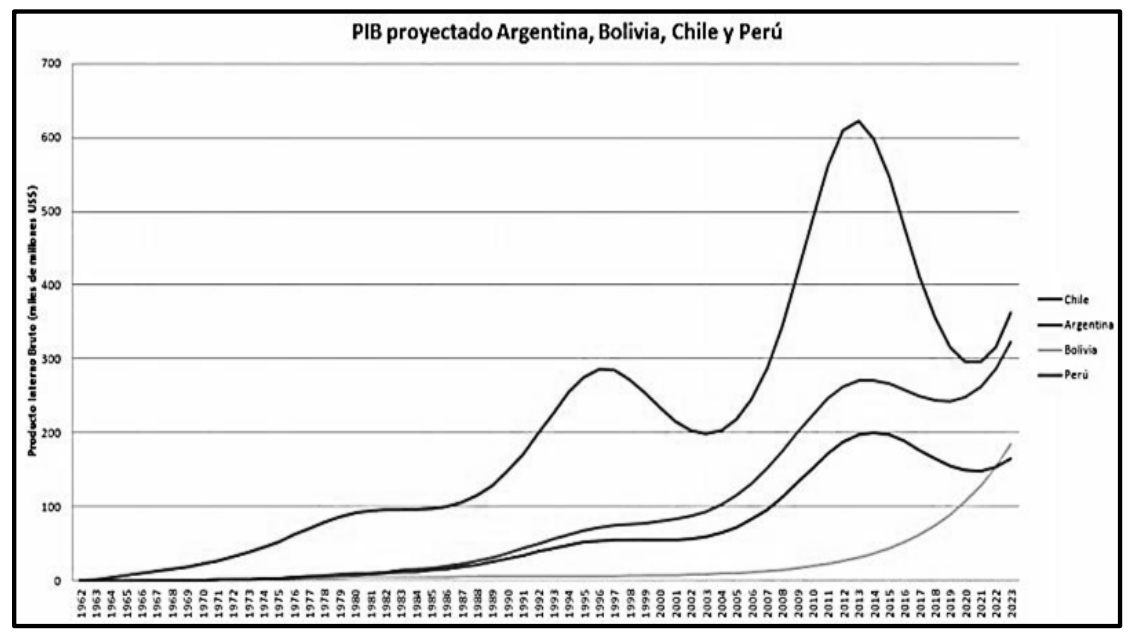

Fuente: Elaboración propia.

En el contexto del análisis de la proyección obtenida en Tabla 6 se presentan una comparación entre los valores del PIB correspondientes al 2015 (CEPALSTAT, 2016) y los proyectados según nuevo modelo propuesto.

Tabla 6

Valor del PIB 2015 (real) y valores proyectados para el mismo año, según modelo elegido

\begin{tabular}{|l|c|c|c|c|}
\hline & Chile & Argentina & Bolivia & Perú \\
\hline CEPALSTAT & 240.796 & 632.84 & 32.998 & 189.209 \\
\hline Modelo Propuesto & 248.437 & 570.046 & 34.930 & 193.3311 \\
\hline
\end{tabular}

Fuente: Elaboración propia.

Como información anexa, se debe tener en consideración que el crecimiento de las economías latinoamericanas han estado sujetas a diversos factores (crisis de deuda externa, crisis asiática de 1997, entre otras) y que han afectado el ciclo económico normal; no se debe descartar los posibles efectos en dichos países que causará el próximo gobierno de los Estados Unidos, mercado que constituye una variable importante en las exportaciones de estas naciones.

Habiendo comentado lo anterior, el PIB chileno y bajo esta proyección (Figura 6), se pronostica que habrá una leve caída para el periodo 2015-2019; 
como está ocurriendo actualmente con el PIB chileno, según información de CEPALSTAT (2016), que presenta como cifras de los últimos años, millones de dólares, 277.078 para 2013, 258.733 para 2014 y para el 2015 se tiene 240.796.

Para Argentina, se tiene que el PIB de los últimos años; según la misma fuente de información, para el 2013 se tiene 613.316, el 2014 presenta como cifra 567.050 y para el 2015 se tiene 632.841; la predicción indica caída brusca, lo que no se estaría dando aunque si existe una disminución desde 2013 al 2014. Habrá que esperar los siguientes años para visualizar el cumplimiento o no de la predicción.

En el caso de Bolivia, se pronostica un crecimiento suave pero sostenido lo que se estaría cumpliendo levemente al cotejarlo con las cifras obtenidas de CEPALSTAT, para 2013 se tiene 30.659, para 2014 corresponde 32.996 y para 2015 se tiene 32.997.

Finalmente, para Perú, los valores reales del PIB de los años 2013, 2014 y 2015 son 201.217, 201.046 y 189.209, respectivamente. Estos valores estarían confirmando, hasta el momento, lo que plantea la curva de predicción.

En síntesis, las predicciones del PIB de las cuatro naciones estudiadas están dentro del margen de lo aceptable pero, claramente, se requieren otros datos futuros para señalar que tan exacta es su anticipación al futuro

\section{CONCLUSIONES}

Como antecedente inicial, es relevante la diferencia de ajuste que presenta el algoritmo Levenberg-Marquardt sobre el exitoso algoritmo Newton-Raphson y el algoritmo Gauss-Newton. Su facilidad de trabajo es de destacar puesto que sólo haciendo uso del Jacobiano de la ecuación a tratar logra una excelente convergencia a la solución igualando en el resultado a Newton-Raphson no requiriendo de las segundas derivadas de la expresión matemática en estudio ni requerir una aproximación cercana al punto de óptimo de solución a los parámetros buscados. En lo referente a convergencia, casi todos los algoritmos incluyendo el implementado por Matlad (función lscurvefit), tienen dificultad cuando los valores iniciales no se acercan a los valores de los parámetros de óptimo buscado; la excepción lo constituye el algoritmo Levenberg-Marquardt que no requiere que se cumple esto en forma estricta, sólo faltará investigar cuál es la tolerancia de distancia de la semilla inicial con respecto a los valores buscados. En particular, la función Iscurvefit mencionada se puede considerar una herramienta de fácil uso y sus resultados son equivalentes a los obtenidos mediante la implementación algorítmica. Su aplicabilidad se potencia al permitir ingresar el modelo sólo considerando su ecuación original.

Desde el punto de vista de la economía, para los modelos, es apreciable el aporte de predicción de la tendencia del comportamiento del crecimiento de la 
economía mediante el análisis del PIB de un país. Como resultado, los modelos analizados permiten obtener una curva de tendencia aproximada que se puede considerar como aceptable. Ahora bien, está claro que un modelo u otro será el adecuado según sea la cuerva del PIB del país en análisis; por ejemplo particular, el modelo Gompertz es adecuado puesto que sus aproximaciones son aceptables.

Otro aspecto importante, es señalar que Gompertz no ha permitido predecir las irregularidades del crecimiento económico de un país (como por ejemplo, principalmente el caso de Argentina), donde las eventualidades intermedias requieren modelos más específicos para tal efecto pero, aún así, se obtuvo una la tendencia aproximada de dicho crecimiento. Una posible estrategia podría ser un ajuste por tramos. Además, la proyección es claramente mejor que la que se obtiene a partir de un modelo lineal de regresión.

Estadísticamente, se genera una correcta visión de la tendencia del PIB pero para obtener una predicción más exacta de un determinado año se deberá considerar alguna estrategia adicional que considere los altos y bajos que se presentan año tras año y que, típicamente, se debe a fenómenos de la contingencia de la economía nacional e internacional de ese momento. Por otra parte, para el modelo Gompertz no es fácil visualizar las tendencias cíclicas que puede presentar el comportamiento del PIB a futuro a excepción de la propuesta del nuevo modelo.

Otra validación que se debe destacar es el hecho que un modelo generado para predecir el comportamiento del crecimiento de entes biológicos también es aplicable para pronosticar el crecimiento de una economía por lo que se concluye que el modelo de Gompertz permite pronosticar, al corto plazo, la tendencia de la misma.

Finalmente, el modelo propuesto presenta mejorías en los indicadores AIC y BIC y representa con mayor claridad el fenómeno de oscilación que presenta los PIB como se señalaba anteriormente pero, aún así, se debe recordar que estas son aproximaciones del comportamiento futuro del crecimiento de una economía, la cual depende de un sinfín de variables tanto endógenas como exógenas de un país. Este modelo presenta la virtud de ajustarse, según sea la curvatura de los datos originales, a la misma tratando de minimizar la distancia entre el dato observado y el estimado; ello, a diferencia del modelo de Gompertz que genera una aproximación sin ajustarse a dichas oscilaciones de la curva original. En síntesis, en este modelo propuesto se tiene que los valores extremos de los datos tratan de ser ajustados de mejor forma a la curva de los datos originales (como se puede visualizar más claramente, por ejemplo, con el PIB de Argentina). 


\section{REFERENCIAS BIBLIOGRÁFICAS}

AGHION, P.; CAROLA, E, \& GARCÍA-PEÑALOSA, C. (1999). "Inequality and Economic Growth: The Perspective of the New Growth Theories". Journal of Economic Literature, Vol. 37, No. 4, Dic.

AKAIKE, H. (1974). "A new look at the statistical model identification". IEEE Transactions on Automatic Control, 19, pp. 716-723.

ALESINA, A. \& RODRIK, D. (1994). "Distributive Politics and Economic Growth". The Quarterly Journal of Economics, Vol.109, No. 2, May.

BARRO, R. (1991). "Economic Growth in a Cross Section of Countries". The Quarterly Journal of Economics, Vol. 106, № 2, May, pp. 407-443.

BARRO, R.J. y LEE, J.W. (2010). "A New Data Set of Educational Attainment in the World, 1950- 2010". NBER Working Paper No. 15902, pp. 1-49.

BERTALANFFY, L. (1957). "Quantitative laws in metabolism and growth". The Quarterly review of Biology, Vol. 32, pp. 217-231.

BURNHAM, K.P. y ANDERSON, D.R. (2002). Model selection and multimodel inference: a practical information-theoretic approach. New York: Springer-Verlag, pp. 488.

CABALLERO, F.F (2011). Selección de modelos mediante criterios de información en análisis factorial. Aspectos teóricos y computacionales. Tesis Doctoral. Granada: 2011.

CASS, D. (1965). "Optimum growth in an aggregative model of capital accumulation". Review of Economic Studies, 32, pp. 233-240.

CEPALSTAT (2016). Base de Datos. http://interwp.cepal.org.

COE, D. y HELPMAN, E. (1993). "International R\&D Spillovers". National Bureau OJ Economic Research Working Paper No. 4444.

COEYMANS, J.E. (1999). "Determinantes de la Productividad en Chile: 1961-1997". Cuadernos de Economía (Latin American Journal of Economics), Instituto de Economía. Pontificia Universidad Católica de Chile., vol. 36(107), pp. 597-637

CORBO, V. y VERGARA, R. (1992). "Los determinantes del crecimiento económico: Introducción". Cuadernos de Economía, Año 29, Agosto, № 87, pp. 165-169.

DRAPER, D. (1995). "Assessment and propagation of model uncertainty". Journal of the Royal Statistical Society, Series B, pp. 196-200.

EDWARDS, S. (1997). "Openness, Productivity, and Growth: What Do We Really Know". National Bureau of Economic Research, (NBER) Working Paper No. 5978, (Cambridge, Massachusetts: MIT Press).

GOMPERTZ, B. (1825). "On the nature of the function expressive of the law of human mortality, and on a new mode of determining the value of life contingencies". Philosophical Transactions of the Royal Society of London, 115, pp.513-583.

HAWTREY, R. y TINBERGEN, J. (1956). El ciclo economico. Hawtrey, Haberler (editor).

HURVICH, C.M. y TSAI, C.L. (1989). "Regression and time series model selection in small samples". Biometrika, 76, pp. 297-307.

HURVICH, C.M. y TSAI, C.L. (1991). "Bias of the corrected AIC criterion for underfitted regression and time series models". Biometrika, 78, pp. 499-509.

KOOPMANS, TJALLING C. (1965). "On the concept of optimal growth". The Econometric Approach to Development Planning. Rand McNally. 
LEE, J. (1995). "Capital goods imports and long-run growth". Journal of Development Economics, Vol. 48, pp. 91-110.

LUCAS, R. Jr. (1988). "On the mechanics of economic development". Journal of Monetary Economics, Elsevier, vol. 22(1), July, pp. 3-42.

MALTHUS, T. (1803). An Essay on the Principle of Population, as It Affects the Future Improvement of Society. London: J. Johnson.

REBELO, S. (1991). "Long-Run Policy Analysis and Long-Run Growth". Journal of Political Economy, University of Chicago Press, vol. 99(3), June, pp. 500-521.

RICARDO, D. (1817). On Principles of Political Economy and Taxation. Kitchener, Ontario, Canada: Batoche Books. 2001.

ROMER, P. (1986). "Increasing Returns and Long-run Growth". Journal of Political Economy, University of Chicago Press, vol. 94(5) Octubre, pp. 1002-37.

SANCHEZ, C. (2013). Estudio probabilistico de modelos de difusión tipo Bertalanffy con funciones terapéuticas. Tesis de Máster en Estadística Aplicada, Universidad de Granada.

SCHUMPETER, J. (1957). La teoría del desenvolvimiento económico: Una investigación sobre ganancias, capital, crédito, interés y ciclo económico. Medellin: Fondo de cultura económica.

SCHWARZ, G. (1978). "Estimating the dimension of a model". The Annals of Statistic, 6(2), pp. 461-464.

SCLOVE, S.L. (1987). "Application of model-selection criteria to some problems in multivariate analysis". Psychometrika, 52(3), pp. 333-343.

SMITH, Adam. (1976) [1776]. An inquiry into the nature and causes of the wealth of nations [WN]. In The Glasgow edition of the works and correspondence of Adam Smith, Vol. 2, eds. R. H. Campbell, and A. S. Skinner. Oxford: Oxford University Press.

SOLOW, R. (1956). "A Contribution to the Theory of Economic Growth". Quarterly Journal of Economics, 70(1), pp. 65-94.

SUGIURA, N. (1978). "Further analysis of the data by Akaike's information criterion and the finite corrections". Communications in Statistic, A7, pp. 13-26.

VERHULST, P.F. (1845). Recherches mathématiques sur la loi d'accroissement de la population. Nouv. Mém. Acad. R. Sci. Lett. B.-Arts Belg. 20, pp. 142-173.

YANG, C. y YANG, C. (2007). "Separating latent clases by information criteria". Journal of Classification, 24(2), pp. 183-203. 